\title{
Ethnic inequalities in severe mental disorders: where is the harm?
}

\author{
James Y. Nazroo ${ }^{1}$
}

Received: 25 May 2015/Accepted: 28 May 2015/Published online: 3 June 2015

(C) Springer-Verlag Berlin Heidelberg 2015

\section{Introduction}

In this issue, Qassem et al. [1] report novel findings on the relative prevalence of psychotic illness for Black (Caribbean and African) and white people in the UK, using data drawn from three large national surveys of psychiatric morbidity covering the period 1993-2007. The key finding, at least for this reader, is that the odds ratio to have a psychotic illness for Black compared with white people is estimated at 2.7 (albeit with relatively wide confidence intervals of 1.3-5.6). This is the key finding, because, although worryingly high, it is considerably lower than estimates of increased rates of incident psychotic illness among Black Caribbean and Black African people in the UK. One recent study estimated these to be nine and six times higher, respectively [2]. Such high estimates are not exceptional; they have been reported consistently in clinical incidence studies since the early 1970s. However, they are a puzzle, because, as noted by the authors of the current paper [1], two other national surveys, both directed by this author, have also produced relatively low estimates of severe psychotic illness in the Black Caribbean population-odds ratios of 1.8 and 2.0 compared with the white population [3, 4].

Not only is there an inconsistency in estimates of risk between clinical incidence studies and community survey studies, but also an inconsistency when considering rates in the UK and those in the Caribbean, with the much higher rates of first diagnosis being only present in the UK [5].

James Y. Nazroo

james.nazroo@manchester.ac.uk

1 ESRC Centre on Dynamics of Ethnicity and Sociology, University of Manchester, Manchester, UK
This suggests that the driver for higher risk (regardless of the extent of that increased risk) is to be found in the postmigration context, a suggestion supported by evidence from other countries.

It is not clear how to make sense of such findings. Almost all of the research undertaken points to the greater risk of psychotic illnesses among Black people in the UK (and elsewhere), but the extent and the origins of this difference are in dispute. Disputes about extent of difference point directly to questions of methodology. So how robust are these findings?

\section{Methodological difficulties}

Any attempt to estimate the prevalence or incidence of rare and difficult to identify conditions in a group that makes up only a small minority of the population raises obvious difficulties. Over more than 20 years, methods have been established for obtaining reliable and valid estimates of the prevalence of psychosis within a defined population. As described in Qassem et al. [1], these rely on the use of screening instruments, validation of diagnoses for a subset of those screened positive, and then using these data to estimate prevalence. As far as can be determined, these are reliable and valid estimates for the defined population, but they have two important drawbacks. First, they are population estimates that at an individual level contain a large degree of uncertainty-the majority of those who screen positive do not, in fact, reach what might be called clinical criteria for a diagnosis. So, prevalence is estimated using probabilistic methods rather than case finding. This means significant measurement error at an individual level and that the estimate of the role of individual risk factors consequently contains considerable uncertainty. Second, 
the population under study is likely to be defined in ways that exclude some of those most at risk of psychotic illness, such as those in prisons, in psychiatric institutions, or who are non-responders to surveys. If there are differential rates of risk in institutionalisation, or survey non-response, across populations, then comparisons across populations will contain meaningful error.

Similarly, estimates using clinical incidence studies have become increasingly robust. Initial concerns about biases in case notes have been addressed by ascertaining caseness using standardised criteria implemented by reviewers blinded to demographic characteristics. Concerns about underestimates of population denominators have been addressed by careful use of population census data. And, although not extensive, what evidence there is suggests that diagnostic categories are valid across ethnic, cultural and language groups. Of course problems remain. Such studies have the assumption that all incident cases of psychotic illness will come to the attention of health services within a short period; and that none of the cases that are identified as incident are in fact recurrent. Neither assumption may hold.

Also, of course, both methods use checklist approaches to case finding with the assumption that psychotic illness is a discrete category.

Importantly, this checklist approach also applies to the assessment of ethnicity. This raises important questions as to what we mean by ethnicity and what our purpose is, when studying ethnic difference [6], and whether our approach to categorisation is too crude. As Qassem et al. acknowledge [1], the Black category they use contains considerable heterogeneity-Caribbean and African, but also within those broad groups there are different countries of origin, migration histories, class positions, generations and increasing numbers of people with mixed ethnic origins. These issues become even more important when we attempt to identify those factors that increase the risk of psychotic illness among those within a broadly defined ethnic category. What is it about membership of such a broad ethnic group that is important for this higher risk, and for the different estimates of risk provided by community and clinical incidence studies?

\section{Why is there an increased risk of psychotic illness among Black Caribbean and Black African people?}

Qassem et al. identify the social and economic disadvantages faced by Black people as at the root of their higher risk of psychotic illness [1]. Although they do not put it in these terms, this points to the ways in which racialised
Black identities increase risk of economic hardship, unemployment, discrimination and harassment. To this we could add the significance of institutional discrimination and increased risk of negative treatment at the hands of the representatives of key institutions, the impact of living in deprived neighbourhoods and in poor-quality housing, the accumulation of these disadvantages and insecurities across a life course, and the impact of this ongoing disadvantage on one's identity. Survey data that have been designed to allow us to address the contribution of such factors identify racially based social and economic disadvantage as the driver of ethnic differences in health, including risk of psychotic illness-be that experiences of racism, discrimination, fear of racism, economic inequality, economic insecurity, area deprivation, or the combination of these [6, 7].

\section{Where is the harm?}

It is worth emphasising that racism is the key issue here. Direct experiences of racism and discrimination not only increase the risk of psychosis for the individual concerned, but also impact on risk for those who may not directly experience racism or discrimination, but nonetheless perceive their identities as under similar threat. In addition, the historic and contemporary climate of racism has generated social and economic inequalities in almost any sphere we care to consider. Addressing contemporary racism is difficult to pursue, particularly in the current UK climate where questions of racial inequality have disappeared from the policy agenda. Redressing the legacies of historic racism is even more challenging.

It is their engagement with policy that is, perhaps, the greatest weakness of Qassem et al.'s paper [1]. Surprisingly, to me at least, the authors use the evidence in their paper to argue for a greater investment in resources for mental health services in areas with a higher proportion of Black Caribbean and Black African people. In this they implicitly follow the arguments of Singh and Burns who move from the assertion that the higher risk of psychosis for Black people is real to the claims that: 'Construing racism as the main explanation for the excess of detentions [under the Mental Health Act] among ethnic minorities adds little to the debate and prevents the search for the real causes of these difference' [8, pp. 649-650], and that coercive treatment should not be seen as punitive, rather it allows the delivery of appropriate services [8]. Indeed, Qassem et al. go on to say that the discrepancy in the estimated increased rate of psychosis derived from community surveys and clinical treatment studies may result from the 'greater requirements from services' for Black people with psychosis [1]. 
Rather than going along with this line of argument, I contend that there are two important policy conclusions to draw from Qassem et al.'s paper. First is that Black Caribbean and Black African people do face an increased risk of psychotic illness and an increased risk that other research has demonstrated is driven by racially based social and economic disadvantage. Second is that there is a significant discrepancy between that increased risk and the much higher risk for Black Caribbean and Black African people of hospital admission and treatment for a psychotic illness. We can speculate as to the reasons for this discrepancy, but it is worth noting Fernando's comment that 'It is in the field of forensic psychiatry that racial injustices and cultural oppression are most acutely felt by black and Asian service users' [9, p. 4]. Here Fernando points to the complex, coercive and adverse pathways into, through and out of care faced by ethnic minority patients-adverse pathways that have been documented and which are visible in everyday psychiatric practice.

Considering these issues from a wider perspective, it is worth interrogating the role of psychiatric institutions and consequent practice within our society and how this sits alongside and interacts with other regulatory and surveillance institutions, most noticeably criminal justice, and the similar position of racialised groups within each of these realms. Whilst the practicing psychiatrist is no doubt doing her/his best for these patients, this practice operates within a particular institutional framework and with access to a limited set of interventions. When we ask 'where is the harm?' we should consider the implications of psychiatric diagnosis and (often aggressive) treatment for the lifecourse of the patient. Does the maxim of 'Do no harm' actually hold?

\section{Implications}

If the conclusions drawn in the preceding section are seriously considered, an obvious question for both research and practice is how and why for a given risk of psychotic illness ethnic minority people have a much higher risk of institutionalisation? This question cannot, in my view, remain unaddressed. But to address it the profession also needs to examine the role it plays in the regulation of the socially disadvantaged, the alternative services and practices that might be considered, and how it relates to other regulatory services and institutions. A time for deep reflection, or to continue on an uneasy path? $[9,10]$.

Conflict of interest The author states that there is no conflict of interest.

\section{References}

1. Qassem T, Bebbington P, Spiers N, McManus S, Jenkins R, Dein S (2014) Prevalence of psychosis in black ethnic minorities in Britain: analysis based on three national surveys. Soc Psychiatry Psychiatr Epidemiol. doi:10.1007/s00127-014-0960-7

2. Fearon P, Kirkbiride JB, Morgan C, Dazzan P, Morgan K, Lloyd T, Hutchinson G, Tarrant J, Fung WL, Holloway J, Mallett R, Harrison G, Leff J, Jones PB, Murray RM (2006) Incidence of schizophrenia and other psychoses in ethnic minority groups: results from the MRC AESOP Study. Psychol Med 36:1541-1550

3. Nazroo J (1997) Ethnicity and mental health: findings from a national community survey. Policy studies Institute, London

4. Nazroo J, King M (2002) Psychosis-symptoms and estimated rates. In: Sproston K, Nazroo J (eds) Ethnic minority psychiatric illness rates in the community (EMPIRIC)—quantitative report. The Stationery Office, London, pp 47-63

5. Hickling FW, Rodgers-Johnson P (1995) The incidence of first contact schizophrenia in Jamaica. Br J Psychiatry 167:193-196

6. Nazroo J (2003) The structuring of ethnic inequalities in health: economic position, racial discrimination and racism. Am J Public Health 93(2):277-284

7. Karlsen S, Nazroo JY, McKenzie K, Bhui K, Weich S (2005) Racism, psychosis and common mental disorder among ethnic minority groups in England. Psychol Med 35(12):1795-1803

8. Singh SP, Burns T (2006) Race and mental health: there is more to race than racism. Br Med J 333:648-651

9. Fernando S (2003) Cultural diversity, mental health and psychiatry. The struggle against racism. Brunner-Routledge, Hove, East Sussex and New York

10. Morgan A (2015) Is psychiatry dying? Crisis and critique in contemporary psychiatry. Soc Theory Health 13(2):141-161 